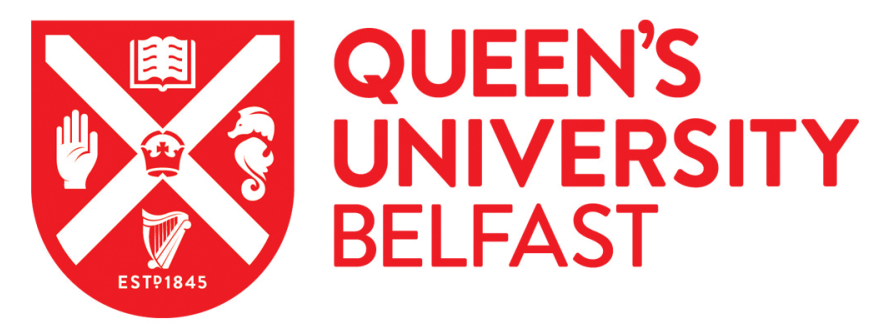

\title{
Use of oximetry to screen for paediatric obstructive sleep apnoea: is one night enough and is 6 hours too much?
}

Galway, N. C., Maxwell, B., Shields, M., \& O'Donoghue, D. (2020). Use of oximetry to screen for paediatric obstructive sleep apnoea: is one night enough and is 6 hours too much? Archives of Disease in Childhood. https://doi.org/10.1136/archdischild-2019-318559

Published in:

Archives of Disease in Childhood

Document Version:

Peer reviewed version

Queen's University Belfast - Research Portal:

Link to publication record in Queen's University Belfast Research Portal

Publisher rights

Copyright 2020 BMJ. This manuscript is distributed under a Creative Commons Attribution-NonCommercial-NoDerivs License

(https://creativecommons.org/licenses/by-nc-nd/4.0/), which permits distribution and reproduction for non-commercial purposes, provided the author and source are cited.

\section{General rights}

Copyright for the publications made accessible via the Queen's University Belfast Research Portal is retained by the author(s) and / or other copyright owners and it is a condition of accessing these publications that users recognise and abide by the legal requirements associated with these rights.

Take down policy

The Research Portal is Queen's institutional repository that provides access to Queen's research output. Every effort has been made to ensure that content in the Research Portal does not infringe any person's rights, or applicable UK laws. If you discover content in the Research Portal that you believe breaches copyright or violates any law, please contact openaccess@qub.ac.uk. 
Title: Use of oximetry to screen for paediatric obstructive sleep apnoea: Is one night enough and is 6 hours too much?

Authors

Niamh C Galway ${ }^{1}$, Barbara Maxwell ${ }^{1}$, Michael D Shields ${ }^{1,2}$, Dara O’Donoghue ${ }^{1,2}$

Authors' Institution

1. Royal Belfast Hospital for Sick Children

2. Queen's University Belfast

Corresponding author's name and address:

Dr Dara O'Donoghue

Centre for Medical Education

Queens University Belfast

Mulhouse Building

Grosvenor Road

BT12 6DP

e-mail: d.odonoghue@qub.ac.uk

Word count of main paper: 2500

\section{Statement author's individual contribution}

NCG: collected the data, analysed the data, co-wrote the paper

BM: collected the data, co-wrote the paper

MDS: helped with study design, analysed the data and co-wrote the paper

DOD: conceived the study, supervised data collection and data analysis, co-wrote the paper. 


\section{ABSTRACT}

\section{Introduction:}

Nocturnal pulse oximetry can be used to screen for obstructive sleep apnoea (OSA) using the McGill Oximetry Score (MOS). The MOS has a time threshold for a technically adequate study of 6 hours. It has been suggested that one night of oximetry is sufficient to screen for OSA using the MOS.

Aims:

1. To evaluate night-to-night variation of the MOS.

2. To determine the impact of recording three nights of oximetry on the screening yield for OSA.

3. To explore whether useful MOS data is discarded when a threshold of six hours of oximetry recording is used.

\section{Methods:}

A retrospective study of nocturnal pulse oximetry done at home over 3 consecutive nights in paediatric patients with suspected OSA. Studies were scored (MOS) using thresholds of $\geq 6$ and $\geq 4$ hours recording.

\section{Results:}

A total of 329 patients were studied. MOS scores over 3 Nights showed only fair to moderate agreement.

On the first night 126 patients (38\%) screened positive for OSA. When three nights of oximetry were done 195 patients (59\%) screened positive on at least one of the nights.

There were 48 patients with studies of between 4-6hours duration on one or more nights. If these studies are scored 20 patients (42\%) would screen positive for OSA on at least one night based on scoring these studies alone.

\section{Conclusion:}

One night of oximetry screening may not be sufficient to screen for OSA. Lowering the time threshold to $\geq 4$ hours may increase the screening capability of nocturnal oximetry. 


\section{Abbreviations}

Apnoea-hypopnoea index (AHI)

Artefact free (AF)

Confidence Intervals (Cl)

Cohen's Kappa $(\kappa)$

Down Syndrome (DS)

Electroencephalogram (EEG)

McGill Oximetry Score (MOS)

Obstructive Sleep Apnoea (OSA)

Oxyhemoglobin saturation measured by pulse

oximetry $\left(\mathrm{SpO}_{2}\right)$

Polysomnography (PSG)

Rapid Eye Movement (REM)

Royal Belfast Hospital for Sick Children (RBHSC)

\section{Key words:}

- Obstructive sleep apnoea

- McGill oximetry score

- Nocturnal pulse oximetry 


\section{INTRODUCTION}

Overnight polysomnography (PSG) in a sleep-laboratory is the gold standard diagnostic tool for obstructive sleep apnoea (OSA). Nocturnal pulse oximetry is an alternative to screen for OSA when PSG is not available and is low cost and easy to use.[1,2] Brouillette et al demonstrated that abnormal oximetry, based on cyclical clusters of oxygen desaturations, has a high positive predictive value but a low negative predictive value for detecting OSA compared with PSG.(3) Nixon et al. subsequently used this to develop the McGill Oximetry Score (MOS) as a classification of the severity of OSA.(4)

A previous study comparing oximetry studies done over two consecutive nights using the MOS showed very good consistency-suggesting that one night of pulse oximetry may be sufficient to screen for OSA.[5] PSG has been shown to have significant night-to-night variation when two consecutive nights were compared.[6,7] This has been termed the 'first night effect'. [8] This may also apply to nocturnal pulse oximetry. Further research using the MOS needs to be done before accepting that a single night study is adequate to screen for OSA.

The MOS was developed to evaluate patients referred for investigation of possible OSA secondary to adenotonsillar hypertrophy. $[4,9]$ It has also been used to evaluate OSA in patients with Down Syndrome (DS).[10] However, there is limited evidence of it being used in other conditions that have an increased incidence of OSA such as Prader-Willi Syndrome and Pierre-Robin Sequence. 
The MOS uses a minimum recording duration of at least 6 hours.[3] Other studies reporting the interpretation of oximetry recordings have used a minimum duration of 5 hours. $[11,12]$ More recently $\geq 3$ hours of artefact free (AF) oximetry has been used to assess myofunctional training on OSA in children with DS.[13] The absolute minimum duration for a sleep recording to get useful data is currently unknown.[14]

Local practice in The Royal Belfast Hospital for Sick Children (RBHSC) has been to perform three consecutive nights of home pulse oximetry to screen for OSA. The aims of this study are:

i) To evaluate the extent of night-to-night variation in the MOS over three consecutive nights in otherwise healthy children and in those with a condition predisposing to OSA.

ii) To determine the impact of recording three nights of oximetry instead of one on the screening yield for OSA.

iii) To explore whether useful MOS data is discarded when a threshold of six hours is used.

\section{METHODS}

\section{Design}

A retrospective observational study evaluating night-to-night variation of at-home nocturnal pulse oximetry over three consecutive nights was conducted. 


\section{Patients and setting}

Patients under 18 years old referred to the paediatric respiratory clinic in RBHSC with clinically suspected OSA between December 2010 and November 2016 were included. Patients were divided into three groups: Group 1 included otherwise healthy children with tonsillar +/adenoidal hypertrophy. Group 2 comprised of children with DS. Group 3 included patients with conditions other than DS that have an increased risk of OSA (e.g. Prader-Willi Syndrome). The study was part of a quality improvement project and ethics committee approval was not required.

\section{Nocturnal pulse oximetry}

Parents attended the RBHSC respiratory clinic and were given a motion-resistant oximetereither the Nonin 9600 Avant Digital Pulse Oximeter (Nonin Medical Inc, Plymouth MN, USA) or the Masimo Radical 5 Pulse Oximeter (Masimo Corporation, Irvine, CA). They were o provided with verbal and written instruction on how to use it for three consecutive nights at home. The sensor probe was to be attached to the child's finger or big toe and they were asked to remove it if the child awakens. Parents were also asked to keep a sleep record documenting the time of sleep onset, morning wake-up time and any night time awakenings. Continuous AF trace that aligned with the sleep record was assumed to be recording sleep. Recordings were analysed using nVision software (Nonin Medical Inc, Plymouth MN, USA) for the Nonin 9600 Avant and Visi-Download (Stowood Scientific,Oxford, UK) for the Masimo Radical 5. Poor perfusion, low signal, and sensor displacement artefact were extracted. Oxygen saturations ( $\mathrm{SpO2}$ ) were averaged over a 
maximum of 3 second increments. Length of time of AF trace (hours) and MOS were recorded. Pulse oximetry studies were independently reviewed by three researchers (NCG, BM, DO'D) and data was entered into an anonymised study database.

The MOS scores the severity of OSA. A positive oximetry recording for OSA has $\geq 3$ desaturation clusters and at least $3 \mathrm{SpO}_{2}$ drops to $<90 \%$ (MOS 2).[3] Increasingly severe OSA is scored as MOS 3 or 4 if there are $\geq 3$ clusters of desaturation and at least $3 \mathrm{SpO}_{2}$ drops to $<85 \%$ and $80 \%$ respectively. If the recording is normal or inconclusive ( $<3$ desaturation clusters and $<3 \mathrm{SpO}_{2}$ drops to $<90 \%$ ) it is scored MOS 1 . A technically adequate pulse oximetry study has been defined as one with $\geq 6$ hours of continuous AF data.[4]

An additional subanalysis of MOS scoring was performed on studies with 4-6 hours of continuous AF data. These studies would otherwise have been rejected as technically inadequate.

\section{Statistical analysis}

Statistical analysis was performed using Stata 15 (Stata Statistical Software: Release 15, StataCorp, College Station, Texas) and StatsDirect V.3 (StatsDirect statistical software, http://www.statsdirect.com). Cohen's Kappa (к) statistic with 95\% Confidence intervals (CI) was used to express the level of agreement between nights with $\kappa>0.6$ indicating good agreement. Quantitative agreement statistics were not calculated given the categorical nature of the MOS. Normally distributed data was expressed as the mean \pm standard 
deviation (SD). Data which was not normally distributed was expressed as the median with interquartile ranges (IQR).

\section{RESULTS}

Nocturnal home oximetry was performed on 329 children (60\% male) with median age 4 years (IQR: 2 to 7 years) in the study time-frame. Group 1 consisted of 204 patients (62\%), Group 2 had 35 (11\%) patients and Group 3 had 82 (25\%) patients. Clinical information was missing from 8 patients and these patients were not assigned a group. There were 126 patients (38\%) with $\geq 6$ hours continuous AF oximetry trace on night 1 who had MOS $\geq 2$ suggesting possible OSA. There were 195 patients (59\%) with $\geq 6$ hours continuous AF oximetry trace (on one or more nights) who had MOS $\geq 2$ on at least one of the nights.

\section{Trace duration}

The mean AF trace duration on Night 1 was 8.7 hours, on Night 2 was 8.1 hours and on Night 3 was 7.8 hours. On Night 1,2 and 3 the number of patients with $<6$ hours AF traces (out of the total 329 patients) were $53(16 \%), 69(21 \%)$ and 76 (23\%) respectively. There were 19 (6\%) patients with $<6$ hours AF tracings on all three nights.

Taking 3 consecutive nights of oximetry reduced the numbers who would have had $<6$ hours AF traces if only one night (taken as the first night) had been performed from $53(16 \%)$ to 19 (6\%). 


\section{Night-to-night variability}

MOS agreement between Nights 1,2 and 3 for the whole study group is tabulated in Appendix 1. Nights 1 and 2, 2 and 3 and 1 and 3 were compared for the whole study group. Patients were excluded from this and subsequent analyses if they did not have technically adequate traces on both nights being compared.

- Comparing Nights 1 and 2, 236 patients showed fair agreement with $\kappa=0.40(95 \% \mathrm{Cl}$ : 0.32 to 0.49$)$.

- Comparing Nights 2 and 3, 223 patients showed fair agreement with $\kappa=0.39(95 \% \mathrm{Cl}$ : 0.31 to 0.48$)$.

- Comparing Nights 1 and 3, 227 patients showed moderate agreement with $\kappa=0.42$ (95\% Cl: 0.34 to 0.51$)$.

\section{Night-to-night variability of Group 1}

There were 204 otherwise healthy patients with tonsillar +/- adenoidal hypertrophy in this group and MOS agreement between Nights 1, 2 and 3 is tabulated in Appendix 1 . Nights 1 and 2,2 and 3 and 1 and 3 were compared.

- Comparing Nights 1 and 2, 152 patients showed moderate agreement with $\kappa=0.41$ (95\% Cl: 0.3-0.52).

- Comparing Nights 1 and 3, 146 patients showed moderate agreement with $\kappa=0.48$ (95\% Cl: 0.37-0.6) 
- Comparing Nights 1 and 3, 142 patients showed fair agreement with $\kappa=0.32(95 \% \mathrm{Cl}$ : $0.21-0.43)$

\section{Night-to-night variability of Group 2}

There were 35 patients with DS in this group and MOS agreement between Nights 1, 2 and 3 is tabulated in Appendix 1. Nights 1 and 2, 2 and 3 and 1 and 3 were compared.

- Comparing Nights 1 and 2, 27 patients showed moderate agreement with $\kappa=0.42$ (95\% $\mathrm{Cl} 0.17$ to 0.66$)$.

- Comparing Nights 1 and 3, 24 patients showed fair agreement between Night 1 and Night 3 with $\kappa=0.36(95 \% \mathrm{Cl} 0.1$ to 0.61$)$.

- Comparing Nights 1 and 3, 26 patients showed moderate agreement with $\kappa=0.47$ (95\% $\mathrm{Cl} 0.24$ to 0.69$)$.

\section{Night-to-night variability of Group 3}

There were 82 patients with other predisposing factors to OSA in this group. A table of the diagnoses and MOS scores is shown in Appendix 2. MOS agreement between Nights 1, 2 and 3 is tabulated in Appendix 1 . Nights 1 and 2, 2 and 3 and 1 and 3 were compared.

- Comparing Nights 1 and 2, 53 patients showed fair agreement with $\kappa=0.31(95 \% \mathrm{Cl}$ 0.14 to 0.5$)$.

- Comparing Nights 1 and 3, 53 patients showed fair agreement with $\kappa=0.27(95 \% \mathrm{Cl}$ 
0.1 to 0.44$)$.

- Comparing Nights 1 and 3, 51 patients showed moderate agreement with $\kappa=0.46$ (95\% $\mathrm{Cl} 0.28$ to 0.64$)$.

\section{MOS scoring of oximetry studies with 4-6 hours AF trace}

There were 48 patients who had at least one oximetry study with 4-6 hours AF recording on at least one of the three nights. If $\geq 4$ hours of AF recording is deemed acceptable then 20 (42\%) of these patients would potentially screen positive for OSA on one night based on MOS scoring of these traces alone. Of these 20 patients; 11 would have an MOS of 2; 4 would have an MOS of 3 and 5 would have an MOS of 4 (Appendix 3). These traces would normally be considered technically inadequate and be rejected. 


\section{DISCUSSION}

The gold-standard investigation to diagnose OSA is PSG but access is limited and hence there is a need to optimise the screening capabilities of other investigations such as pulse oximetry. This study has shown that there is significant variability in the night-to-night pulse oximetry recordings of children when using the MOS to screen for OSA. Paediatric PSGs have been shown to exhibit night-to-night variability in sleep architecture with patients having more rapid eye movement (REM) sleep on Night 2 than Night 1.[6] OSA predominantly occurs in children during REM sleep and a susceptible patient is more likely to have the REM-related cyclical desaturations associated with OSA on subsequent nights rather than the first night.[6,7]. A previous study comparing two consecutive nights of oximetry in otherwise healthy children reported very good 'consistency'.[5] However, this study analysed the correlation rather than the agreement between nights.

We have demonstrated that, by using three consecutive nights of pulse oximetry instead of one, there is a reduction from $16 \%$ to $6 \%$ of patients having no recordings of adequate duration for analysis. In addition, $59 \%$ of patients screened positive for OSA on at least one of the three nights compared with $38 \%$ if only one night had been performed. Local practice has been to perform three consecutive nights of home oximetry to reduce the chances of having to do repeat studies if there was insufficient data on the first night. However, if oximetry is abnormal on the first night there is no requirement to do further recordings on subsequent nights. To streamline this decision-making, it is anticipated that the development of Bluetooth oximeters, with the ability to upload overnight studies to a cloud-based repository using a smartphone, will allow healthcare professionals to view recordings 
remotely on completion of the first night.[15] A decision could then be made on whether further nights are required.

Children with DS have an increased risk of OSA with a reported prevalence between $31-79 \%$. [16-18]. The MOS has been shown to be applicable to patients with DS. [10] Indeed, a systematic review has suggested that nocturnal pulse oximetry may be the preferred method for diagnosing OSA in DS patients as they are more likely to display limited cooperation with PSG. [19] In the present study, fair to moderate agreement in the MOS was shown over three consecutive nights in patients with DS.

The agreement in MOS between nights for patients with co-morbidities that may make them more susceptible to OSA (Group 3) showed fair to moderate agreement. Interestingly, a recent study suggests that the positive predictive value of the MOS may be lower in this group due to non-obstructive conditions causing desaturations.[20]

The lower time threshold required to score nocturnal pulse oximetry tracings has been taken as 6 hours.[4] It has been recognised that further work needs to be done to determine the minimum recorded sleep time for children. [14] In the present study, reducing the threshold duration for technically adequate oximetry traces to a minimum of 4 hours increased the number of patients with an MOS $\geq 2$ who would have been screened positive for OSA. As the specificity of the MOS as a screening test for OSA is high, this may suggest that an abnormal study (MOS $\geq 2$ ) could be considered as being positive for OSA. However, as the sensitivity of the MOS is limited, a study of 4-6 hours with a MOS 1 would not provide any additional useful information. 
There are a number of limitations to this study. It is a retrospective study and it is recognised that further prospective studies are needed to confirm the study findings. The averaging time of the Masimo Radical 5 pulse oximeter is 2 seconds and the averaging time of the Nonin 9600 Avant Digital Pulse Oximeter is 3 seconds. The pulse oximeters were used in approximately equal numbers. Ideally all patients would have been studied using oximeters with the shorter averaging time. It is known that using oximeters with longer averaging times may underestimate the number of desaturations.[21] However it is unlikely that a 1 second difference in the averaging times will affect the MOS score. Indeed, a comparative study of two oximeters with different averaging times, suggested that even a 4 second difference did not affect the classification of severity of OSA [22].

Some of the patients in this study had been included in a previous study. [23] However no MOS data on any of these patients has previously been reported. 


\section{CONCLUSION}

This study on the use of the MOS to screen for OSA in children suggests that, when it is repeated on three consecutive nights, there is only fair to moderate agreement between MOS scores and that, if only one night of oximetry is performed, the screening yield may be limited. The study also found that OSA can be detected using the MOS in studies of between 4 and 6 hours duration. In order to optimise nocturnal oximetry as a screening test for OSA we suggest that more than one night of nocturnal at home pulse oximetry should be done and that studies $\geq 4$ hours duration should be scored. 


\section{What is already known on this topic?}

1. Nocturnal pulse oximetry is an alternative to screen for OSA when PSG is not available.

2. It has been suggested that one night of nocturnal pulse oximetry is sufficient in order to diagnose OSA.

3. The MOS is a recognised score for estimating the severity of OSA based on nocturnal pulse oximetry but requires a minimum of 6 hours recording duration.

\section{What this study adds?}

1. There is significant night to night variability in screening for OSA using nocturnal pulse oximetry.

2. Performing three nights of oximetry instead of one increases the screening yield for OSA.

3. OSA can be detected in studies of between 4- 6-hours duration. 


\section{References:}

1. Marcus CL, Brooks LJ, Draper KA, et al. Diagnosis and management of childhood obstructive sleep apnea syndrome. Pediatrics 2012; 130 (3):576-84

2. Kaditis AG, Alanso Alvarez ML, Boudewyns A, et al. Obstructive sleep disordered breathing in 2- to 18-year-old children: diagnosis and management. European Respiratory Journal 2016; 47(1):69-94

3. Brouillette RT, Morielli A, Leimanis A, et al. Nocturnal pulse oximetry as an abbreviated testing modality for pediatric obstructive sleep apnea. Pediatrics 2000; 105(2):405412

4. Nixon GM, Kermach AS, Davis GM, et al. Planning adenotonsillectomy in children with obstructive sleep apnea: the role of overnight oximetry. Pediatrics 2004; 113:e19-25

5. Pavone $M$, Cutrera R, Verrillo $E$, et al. Night-to-night consistency of at-home nocturnal pulse oximetry testing for obstructive sleep apnea in children. Pediatric Pulmonology 2013; 48(8):754-760

6. Scholle S, Scholle HC, Kemper A, et al. First night effect in children and adolescents undergoing polysomnography for sleep-disordered breathing. Clinical Neurophysiology 2003; 114(11):2138-45

7. Verhulst SL, Schrauwen N, De Backer WA, et al. First night effect for polysomnographic data in children and adolescents with suspected sleep disordered breathing. Archives of Disease in Childhood 2006; 91(3):233-7

8. Agnew HW Jr, Webb WB, Williams RL. The first night effect: an EEG study of sleep. Psychophysiology 1966; 2(3):263-266

9. Horwood L, Brouillette RT, McGregor CD, et al. Testing for pediatric obstructive sleep apnea when health care resources are rationed. JAMA Otolaryngology Head Neck Surgery 2014; 140(7):616-23

10. Coverstone AM, Bird M, Sicard $M$, et al. Overnight pulse oximetry for evaluation of sleep apnea among children with trisomy 21. Journal of Clinical Sleep Medicine 2014; 10(12):1309-15

11. Urschitz MS, Wolff J, Von Einem V, et al. Reference values for nocturnal home pulse oximetry during sleep in primary school children. Chest 2003; 123(1):96-101

12. Velasco Suarez CT, Figueroa Turienzo JM, Len F, et al. Pulse oximetry recording in children with adenotonsillar hypertrophy: usefulness in the diagnostic of obstructive sleep apnea syndrome. Arch Argent Pediatr 2013; 111(3):196-201

13. Von Lukowicz M, Herzof N, Ruthardt S, et al. Effect of a 1-week intense myofunctional training on obstructive sleep apnoea in children with Down Syndrome. Archives of Disease in Childhood 2019; 104:275-279

14. Evans HJ, Karunatilleke AS, Grantham-Hill S, et al. A cohort study reporting normal oximetry values in healthy infants under 4 months of age using Masimo technology. Archives of Disease in Childhood 2018; 103(9):868-872

15. Xu Z, Gutierrez-Tobal GC, Wu Y, et al. Cloud algorithm oximetry-based diagnosis of obstructive sleep apnoea in symptomatic habitually snoring children. European Respiratory Journal 2019 Feb 21;53 (2)

16. Stebbens VA, Dennis J, Samuels MP, et al. Sleep-related upper airway obstruction in a cohort with Down's syndrome. Archives of Disease in Childhood 1991; 66(11):13331338 
17. Hill C, Evans $H$, Gringras $P$, et al. Original Article: Prevalence and predictors of obstructive sleep apnoea in young children with Down Syndrome. Sleep Medicine 2016; 27-28:99-106

18. P Dyken ME, Lin-Dyken DC, Poulton S, et al. Prospective polysomnographic analysis of obstructive sleep apnea in Down Syndrome. Archives Pediatric Adolescent Medicine 2003; 157(7):655-660

19. Kaditis A, Kheirandish-Gozal L, Gozal D. Paediatric OSAS: oximetry can provide answers when polysomnography is not available. Sleep Medicine Reviews 2016; 27:96-105

20. Trucco, Rosenthal M, Bush A, et al. The McGill score as a screening test for obstructive sleep disordered breathing in children with co-morbidities. Sleep Medicine 2020 April; 68:173-176

21. Farré R, Montserrat JM, Ballester $E$, et al. Importance of the pulse oximeter averaging time when measuring oxygen desaturation in sleep apnea. Sleep 1998 June 15; 21(4): 386-90

22. Doorduin J, Eijsvogel M, de Jongh. Pulse Oximeter Averaging Time; Effect On the classification of obstructive sleep apnea-hypopnea syndrome (OSAHS). American Journal of Respiratory and Clinical Care Medicine 2010:181:A6754 https://doi.org/10.1164/ajrccm-conference.2010.181.1 MeetingAbstracts.A6754

23. Burke RM, Maxwell $B$, Hunter $C$, et al. Night-to-night variation of pulse oximetry in children with sleep-disordered breathing. Archives of Disease in Childhood 2016; 101(12):1095-1099 


\section{Licence statement}

* I, the Submitting Author has the right to grant and does grant on behalf of all authors of the Work (as defined in the below author licence), an exclusive licence and/or a non-exclusive licence for contributions from authors who are: i) UK Crown employees; ii) where BMJ has agreed a CC-BY licence shall apply, and/or iii) in accordance with the terms applicable for US Federal Government officers or employees acting as part of their official duties; on a worldwide, perpetual, irrevocable, royalty-free basis to BMJ Publishing Group Ltd (“BMJ") its licensees and where the relevant Journal is co-owned by BMJ to the co-owners of the Journal, to publish the Work in Archives of Disease in Childhood and any other BMJ products and to exploit all rights, as set out in our licence.

The Submitting Author accepts and understands that any supply made under these terms is made by BMJ to the Submitting Author unless you are acting as an employee on behalf of your employer or a postgraduate student of an affiliated institution which is paying any applicable article publishing charge ("APC") for Open Access articles. Where the Submitting Author wishes to make the Work available on an Open Access basis (and intends to pay the relevant APC), the terms of reuse of such Open Access shall be governed by a Creative Commons licence - details of these licences and which Creative Commons licence will apply to this Work are set out in our licence referred to above. 